Voix et Images

voixetimages

\title{
La blessure du temps : mémoire et culture pour Fernand Dumont
}

\section{Éric Méchoulan}

Volume 27, numéro 1 (79), automne 2001

Fernand Dumont

URI : https://id.erudit.org/iderudit/201580ar

DOI : https://doi.org/10.7202/201580ar

Aller au sommaire du numéro

Éditeur(s)

Université du Québec à Montréal

ISSN

0318-9201 (imprimé)

1705-933X (numérique)

Découvrir la revue

Citer cet article

Méchoulan, É. (2001). La blessure du temps : mémoire et culture pour Fernand Dumont. Voix et Images, 27(1), 35-47. https://doi.org/10.7202/201580ar
Résumé de l'article

Par l'analyse des concepts de mémoire, de tradition et de culture, il s'agit de voir apparaître chez Fernand Dumont une pensée politique du temps, dont on trouve les soubassements ou les correspondances chez certains philosophes contemporains. La notion même de blessure permettant d'allouer un tour sensible à cette pensée du temps. d'utilisation que vous pouvez consulter en ligne.

https://apropos.erudit.org/fr/usagers/politique-dutilisation/ 


\title{
La blessure du temps: mémoire et culture pour Fernand Dumont
}

\author{
Eric Méchoulan, Université de Montréal
}

\begin{abstract}
Par l'analyse des concepts de mémoire, de tradition et de culture, il s'agit de voir apparaîtré chez Fernand Dumont une pensée politique du temps, dont on trouve les soubassements ou les correspondances chez certains philosophes contemporains. La notion même de blessure permettant d'allouer un tour sensible à cette pensée du temps.
\end{abstract}

Il y a des esprits qui misent sur le langage, mais il en est d'autres qui sont sensibles d'emblée aux insuffisances et aux leurres de ce langage qu'ils n'en aiment pas moins pourtant - étant peut-être même de ceux qui l'affectionnent le plus, présence blessée, précaire. Et pour ma part je crois que c'est seulement quand on s'attache à lui, et à sa parole, de cette seconde façon, avec soupçon, sentiment de l'exil au sein des mots, et donc nostalgie, élan de tout l'être, exigence, que l'on accède à un sentiment de la finitude qui ouvre - et c'est alors la poésie même - à la mémoire de l'immédiat et à l'expérience de l'unité

Yves Bonnefoy, Lieux et destins de l'image

La blessure est ce qui nous fonde: ni le néant des ciels sereins, ni la plénitude mélancolique des mots d'amour ne suffiraient à faire de nos existences des valeurs si le temps, impatiemment, n'incisait la peau du quotidien et ne révélait, dans l'instant de la douleur, la promesse d'une cicatrice. $\grave{A}$ toute origine, il faut une trace, sans quoi elle ne pourrait passer pour une mémoire ou ne saurait devenir un destin. La blessure, même lentement refermée, conserve encore l'image d'une ancienne ouverture. Car là réside sa 
tâche: ouvrir ce qui semblait clos, continu, uniforme. La plaie témoigne d'un écart des chairs, d'une mise à distance de ce qui formait un seul tissu, au point d'apercevoir alors tout ce qui en compose l'identité: "compose" et non "composait", l'identité ne préexiste pas au regard qui la découvre. La blessure est cette rencontre énigmatique, ce fragile espace où émergent du même mouvement et, par leur articulation même, par leur soudain désordre, le visible de la chair cachée et l'invisible du temps qui fuit.

Entre la perception continue de l'existence et sa suspension ou sa rupture, surgit un "défi " où la pensée de Fernand Dumont trouve, en même temps qu'un héritage évident, son plus fondamental stimulant. Quand il reprend l'élan des méditations cartésiennes, cherchant dans le mouvement de l'attention à soi, dans le geste de la réflexivité, l'ancrage commun pour qui entend trouver une certitude au plus profond de la conscience et pour qui veut appréhender la machinerie sociale de la culture, Fernand Dumont replie dans une brève note de bas de page la perspective bergsonienne du "moi qui dure" sur l'expérience du cogito ${ }^{1}$. Au sens strict, ce serait une bévue conceptuelle si l'on n'y discernait la marque souterraine et l'indispensable relais de la Critique de la raison pure: dans le $₫ 25$ de l'Analytique transcendantale, Kant repère bien le défaut du "je pense" cartésien, incapable de déterminer le "je suis", puisqu'il lui manque la forme pure du temps qui autorise son intuition ${ }^{2}$. Mais c'est, du coup, ainsi que le remarque Gilles Deleuze (de façon, d'ailleurs, exactement contemporaine aux propos de Dumont), faire du cogito "l'affection d'un moi passif qui sent que sa propre pensée, sa propre intelligence [...] s'exerce en lui et sur lui, non pas par lui [...], le JE est comme traversé d'une fêlure: il est fêlé par la forme pure et vide du temps ${ }^{3}$.

L'intuition de Fernand Dumont porte justement sur cette nécessité d'une expérience temporelle de la réflexivité qui lui permet de rabattre la pensée de Bergson sur celle de Descartes et de souligner la dimension fondamentale de cet "être-affecté" qu'il nomme diversement "fissure", "plaie", "déchirement" ou "blessure ". Par exemple: "S'il est, en définitive, quelque caractéristique essentielle de la culture actuelle, elle semble

1. Fernand Dumont, Le lieu de l'bomme. La culture comme distance et mémoire, présentation de Serge Cantin, Montréal, Bibliothèque québécoise, 1994 [1968], p. $72-73$.

2. "Le "je pense" exprime l'acte qui détermine mon existence. L'existence est donc déjà donnée par là, mais la manière dont je dois la déterminer, c'est-à-dire poser en moi le divers qui appartient à cette existence, ne l'est pas encore. Il faut pour cela l'intuition de soi-même qui a pour fondement une forme donnée a priori, c'est-à-dire le temps." (Emmanuel Kant, Critique de la raison pure, trad. A. Tremesaygues et B. Pacaud, Paris, Presses universitaires de France, 1986, p. 136)

3. Gilles Deleuze, Différence et répétition, Paris, Presses universitaires de France, 1989 [1968], p. 116-117.

4. Micheline Cambron avait déjà finement remarqué l'importance de ces "quasi-concepts " pour le travail de Fernand Dumont. Voir sa préface à la réédition de Fernand Dumont, Le sort de la culture, Montréal, l'Hexagone, 1995, p. 10. 
résider dans le sentiment d'un déchirement irréductible entre le monde du sens et celui des formes concrètes de l'existence." Ou encore: "Par l'abolition momentanée des rapports de la conscience et du monde, une autre conscience et un autre monde peuvent surgir, à qui il suffira de devenir poème, tableau ou roman pour qu'ils opposent, et pour toujours, aux bruits familiers de la conscience mondaine, leur troublant et énigmatique défi, leur inguérissable blessure ${ }^{5}$."

On pourrait demeurer surpris de noms aussi profondément investis d'un pathos peut-être inutile: même si l'on parlait dans ces mêmes années soixante de "coupure épistémologique", les noms choisis avec soin par Fernand Dumont semblent chargés d'un inhabituel frisson; noms qui visent, en fin de compte, à rendre sensible une émotion là où le plus neutre et plus habituel terme de "distance" lui suffit parfois :

En même temps que s'explicite la signification du monde dans une culture soucieuse de ses intentions et de ses fondements, la distance se fait plus nette entre elle et ce que, d'une manière confuse, on appelle la vie quotidienne.

La culture est, pour l'homme, distance de soi-même à soi-même.

[Avec le langage] je reprends aussi à mon compte une certaine distance entre un sens premier du monde disséminé dans la praxis propre à mon contexte collectif et un univers second où ma communauté historique tâche de se donner, comme horizon, une signification cohérente d'elle-même. Cette distance et les deux pôles qui l'indiquent, c'est bien ce qu'il faudrait entendre par le concept de culture ${ }^{6}$.

Distance de la culture, en tant que productrice de significations, avec la vie quotidienne; distance qui creuse le rapport de tout être avec soimême; distance qui ordonne, en fait, la notion de culture. Ces variations indiquent combien la culture est loin de se limiter à ce réservoir d'identités toutes préparées, à ce stock de pratiques inconsciemment assimilées ou à cet ensemble d'œuvres où se réfléchit le monde des hommes, car elle est d'abord le lieu d'une épreuve. C'est pourquoi il faut aussi dire cette distance sous la forme plus sensible du déchirement ou de la blessure. Il ne faut pas y voir l'élégant decorum du poète, mais le souci proprement littéraire de nouer dans les mots les matérialités conceptuelles et les rythmes des idées.

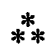

On doit reconnaître à cette sensibilité la vertu du drame, à la fois par le vertige d'une expérience qui touche, voire qui entame le corps lui-

5. Fernand Dumont, Le lieu de l'bomme, op. cit., respectivement p. 27 et p. 84 . Je souligne.

6. Ibid., respectivement p. 26, p. 30 et p. 62. 
même, et par le jeu des rôles qu'il implique. Sans doute l'examen de la modernité à partir de son plus ancien adversaire, la tradition, induit-il chez Dumont la possibilité d'en traiter en termes de scénarios et de rôles et de révéler, par là même, la secrète parenté de ces deux manières ou de ces deux époques. La société traditionnelle voue, en effet, les êtres qui la composent à recevoir les rôles qu'ils auront à jouer selon leurs états, leurs fonctions, leurs âges, leurs sexes et les situations contingentes dans lesquelles ils se trouvent pris. Le sentiment moderne par où le sujet se façonne lui-même, seul, face à d'autres êtres tous également producteurs de leurs styles propres, se trouve, dès lors, reconduit mais déplacé. On y reconnaît encore des volontés d'édifier son habitat, mais dans un milieu et avec des matériaux reçus et assimilés. Réciproquement, la tradition apparie le nouveau aux vestiges de l'ancien (il suffit à la création de prendre le masque sacré du précédent ou de l'immémorial). De cette dichotomie, Fernand Dumont tire la leçon d'une équivoque continuité, comme, dans la plaie, "la faille et la masse entamée sont à la fois en relation d'opposition et de complémentarité ${ }^{7}$ ".

Au découpage de la tradition et de la modernité, dans la longue histoire des sociétés humaines, se superposent deux cultures que tout oppose et relie: culture vécue ou dispersée, culture prescrite ou institutionnalisée - ou, plus généralement, culture première et culture seconde, ainsi qu'il les nomme. La première suppose un sentiment du commun et un exercice du familier où s'associent sens vécu et sens aperçu pour l'ensemble d'un groupe; la seconde s'appuie sur le changement et la recherche d'une cohérence des significations à partir d'une dissociation entre sens vécu et sens aperçu. En celle-là, on reçoit du monde le sens; en celle-ci, on produit le sens du monde. Ici, on quête un idéal qui ressortit alors de l'avènement; là, on recherche l'intégration et la production des fins d'un événement.

On peut dire, en définitive, que la culture vécue court sur le fond d'une signifiance généralisée; face à tout ce qui arrive, les êtres se demandent : de quoi est-ce le signe? Au contraire, la culture prescrite repose sur un fond d'insignifiance générale; devant ce qui arrive, on se demande: en quoi est-ce un signe? C'est pourquoi, autant cette culture première densifie la durée, en valorisant la répétition, autant la culture seconde étale la durée en estimant les répétitions insupportables.

Les formes historiques que peut prendre cette dichotomie n'empêchent cependant pas qu'elle traverse en fait toute société, même si une différence au moins persiste: dans les sociétés traditionnelles, la culture ne forme pas de sphère particulière, tandis que, dans les sociétés modernes, elle fait l'objet d'interprétations spécifiques qui en déréalisent le

7. Micheline Cambron, "Préface ", Le sort de la culture, op. cit., p. 10. 
vécu et en valorisent la représentation. De ce point de vue, la dissociation entre culture d'élite et culture populaire ne décrit pas tant deux formations culturelles que la construction par la culture savante, sous la forme d'une histoire et d'un progrès, du passage du populaire à l'élite. La culture savante se compose et se valorise elle-même en construisant, comme son autre, la culture populaire: "La culture savante a ceci de singulier qu'elle est une reprêsentation de la culture. La culture barbare, sauvage, populaire est son opposé, pour mieux dire son résidu; elle peut donc être son objet $^{8}$."

Si l'on poursuit encore le jeu d'opposition entre le vécu et l'institutionnalisé, on s'aperçoit que la tradition renvoie au passé, alors que l'histoire le raconte: "La science historique que nous connaissons n'est pas le prolongement de la tradition; elle est son renversement. La tradition renvoie au passé; elle ne le raconte pas ${ }^{9}$." D'un côté, témoignage qui répond à un souci de légitimation et d'intelligibilité du présent; de l'autre, trace qui rend intelligible le passé, devenu énigmatique depuis que le présent en est coupé. De part et d'autre, c'est encore de pouvoir qu'il s'agit. En rejetant dans le passé les modèles dont il faut alors hériter, on place le pouvoir hors de portée des êtres vivants qui composent l'ensemble social: le passé a pour charge de maîtriser le présent. À l'inverse, en faisant du passé une altérité par rapport au présent et du présent la source d'innovations constantes, on positionne le pouvoir dans l'invention de soi, au point que, désormais, le présent domine le passé (par ces experts en souvenirs que sont les historiens). Mais en élisant certains moments du passé pour y chérir des avènements, la tradition favorisait aussi l'oubli massif de nombre d'événements. En allouant, au contraire, tant de poids aux événements, les sociétés modernes ne savent plus se débarrasser des innombrables souvenirs qui pourraient devenir signifiants, mais que l'on perd dans la masse documentaire.

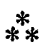

Dans quelle configuration sociale la distance trouve-t-elle sa plus grande évidence? On pourrait croire que, dans les sociétés modernes où les individus sont séparés les uns des autres, où les êtres se trouvent dépossédés de leurs productions, où le passé est coupé du présent, où la culture seconde s'est particulièrement développée, la mise à distance joue plus volontiers que dans les sociétés traditionnelles. Rien n'est moins sûr, car le sentiment de distance du groupe face à son passé ou à son destin collectif trouve dans la dette sa figure la plus énigmatique. Par définition,

8. Fernand Dumont, Le sort de la culture, op. cit., p. 150-151. Je souligne.

9. Id., L'avenir de la mémoire, Québec, Nuit blanche, 1995, p. 25. 
la tradition est d'abord transmission, autrement dit, héritage. Identités, pratiques, rituels, savoirs proviennent tous d'ancêtres ou de dieux du passé envers lesquels on ne peut manquer de demeurer en dette ${ }^{10}$. Tel est ce que les sociétés modernes n'ont eu de cesse d'effacer, en tentant de se donner à elles-mêmes leurs propres lois, sans égard pour la valeur de l'héritage.

Or c'est bien en termes d'héritage que Fernand Dumont entend définir la culture, toute culture: "C'est grâce à la culture que l'humanité se déprend de la répétition monotone à laquelle est vouée la condition animale, qu'elle s'inscrit dans une histoire où ses actions se prêtent à une accumulation des œuvres et à un surplomb du devenir. La culture est donc un héritage. Voilà en quoi elle pose un problème de mémoire ${ }^{11}$." Les sociétés modernes n'y échappent pas; elles ont simplement tâché d'oublier la dette structurelle qui ne pouvait manquer de les édifier, en faisant du passé un poids plutôt qu'un appui.

On ne peut manquer d'être frappé aujourd'hui par l'effervescence qui touche la mémoire: elle est partout. Engouement pour les commémorations, fascination pour le patrimoine, retour ritualisé des modes d'antan, valorisation des traditions et des folklores; la mémoire est devenue un spectacle. Aux révolutions des temps modernes, et à l'empire de la notion même de révolution, succèdent aujourd'hui la restauration de la mémoire et la tâche du souvenir. Si tâche il y a, c'est que la mémoire s'est perdue. Nous visitons aujourd'hui volontiers notre passé parce que la fée de l'histoire l'a changé en musée. Le passé ne se projette plus dans le présent pour l'irriguer de sès leçons, comme avec la tradition, il en est irréductiblement séparé pour mieux être examiné : le passé est objet de connaissance plutôt que réservoir de savoirs.

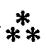

Fernand Dumont voudrait bien prendre à rebrousse-poil le sens de l'histoire et transformer le spectacle de la mémoire en identité collective. Non par vœu pieux, mais par sens du politique. Comme il le signale, en effet, la production de normes (au lieu de leur réception dans la tradition) nous oblige à recourir au droit plutôt qu'à l'éthique :

Par suite de la pluralité, de la relativité, de l'usure rapide des normes de conduite, les accords spontanés le cèdent, en bien des secteurs de la vie, à des règles formelles justifiées autrement que par la longévité de leurs applications. La production de la norme, bien plus que sa réception, s'accompagne d'un recul de l'éthique au profit du droit. L'éthique fait d'abord appel à des

10. Sur cette logique de la dette, je me permets de renvoyer à mon article "Dette, institution, histoire ", Surfaces, vol. VI, 1997.

11. Fernand Dumont, L'avenir de la mémoire, op. cit., p. 17-18. 
attitudes qui s'expriment sans qu'on doive s'interroger sans cesse sur leur fondement; tandis que le droit provient d'un départage où la raison se combine à la force pour imposer ses diktats ${ }^{12}$.

Cette ultime remarque sonne très pascalienne, même si Fernand Dumont n'en conserve pas l'inquiétante tension: "Ne pouvant faire qu'il soit forcé d'obéir à la justice, on a fait qu'il soit juste d'obéir à la force. Ne pouvant fortifier la justice, on a justifié la force, afin que le juste et le fort fussent ensemble ${ }^{13}$." Le parallèle avec Pascal ne porte pas seulement sur les hasards d'une référence, mais sur une commune entreprise d'adhésion à la tradition (elle-même entée sur une éthique chrétienne).

Si Fernand Dumont oppose, en effet, dans L'avenir de la mémoire, sociétés traditionnelles et modernes, c'est pour mieux essayer de sauver la notion de tradition. La tradition ne serait que la "raison d'être ${ }^{14}$ " des coutumes. Une fois celles-ci disparues dans la modernité industrielle et urbaine, le passé devient une énigme et l'avenir un projet: se souvenir est alors un métier, celui de l'historien. Mais l'histoire n'est pas seulement connaissance de l'autrefois, travail documentaire où le présent se met à distance du passé, elle est aussi valorisation, appropriation du passé, œuvre de témoignage possible par où le passé s'insère dans le présent. Fernand Dumont en fait deux lectures des traces du temps "comme des documents à propos desquels je peux m'interroger: cela s'est-il déroulé tel qu'on le rapporte, dans quelle mesure puis-je m'y fier? Les traces du passé, il m'est loisible aussi d'y voir des témoignages, dont je me sente solidaire sans nécessairement m'y identifier tout à fait, et dont je témoignerai à mon tour ${ }^{15}$."

Dans nos sociétés régies par la production où les sujets semblent dépossédés de leur capacité d'agir devant l'autonomie des savoirs et des techniques, le sens de la tradition éviterait les replis dramatiques sur le seul privé: la politique serait moins un spectacle de l'éphémère qu'une participation, une mémoire communément partagée, non simplement reçue, mais à "restaurer sans cesse". Face au pouvoir anonyme de sociétés de plus en plus homogènes, la mémoire comme faculté d'interprétation du quotidien permettrait de nouer un dialogue des traditions, y compris religieuses, en vue d'une nouvelle tradition à l'échelle de l'humanité. Pour y parvenir, il faudrait un souci pédagogique, où l'école soit lieu de savoirs et de valeurs, et un souci politique, où la démocratie puisse susciter de nouvelles idéologies porteuses de plus d'espérance. Comme "il n'y a pas

12. Ibid., p. 49.

13. Blaise Pascal, Pensées, Michel Le Guern (éd.), Paris, Gallimard, 1977, p. 76.

14. Fernand Dumont, L'avenir de la mémoire, op. cit., p. 20.

15. Ibid., p. 58. On pourrait rapprocher cette réflexion de Dumont de celle de Paul Ricœur qui ajoute au modèle de l'empreinte, favori de l'histoire, celui du témoignage, lieu de mémoire ("La marque du passé ", Revue de métaphysique et de morale, mars 1998, p. 732). 
de valeurs sans croyance", la mémoire spirituelle aiderait à fonder les valeurs de la tradition, et la croyance en la démocratie se situerait avant même toute adhésion à telle ou telle idéologie.

On discerne, dans ces remarques de Fernand Dumont, la tentative d'allier les deux dispositifs temporels de la tradition et de la modernité: l'héritage et le projet, la réception et la production: "La culture est à la fois un legs qui nous vient d'une longue histoire et un projet à reprendre; en un certain sens, elle n'est rien d'autre qu'une mémoire ${ }^{16}$. "Loin, donc, de s'opposer à la mémoire, la culture ne devrait en être que le prolongement ou le pendant.

***

Pourtant ce n'est qu'" en un certain sens " que la culture est mémoire. On doit, en effet, se souvenir que la culture relève de procédures et de dispositifs essentiellement modernes. On peut, certes, arguer que la culture renvoie à tout stock de savoirs, d'expériences et de pratiques qui constituent les sociétés humaines: en ce sens là, il est clair que chaque groupe possède sa propre culture. Mais il faut aussi remarquer combien cette notion de culture est une production occidentale et moderne (a fortiori les découpages savants de la culture savante et de la culture populaire: au sens strict, comme on l'a vu, il y a bien des traditions populaires, mais sans doute pas de "culture populaire", sinon soigneusement bâtie par la culture savante comme son objet). Avant les $\mathrm{XVII}^{\mathrm{e}}-\mathrm{xv} \mathrm{III}^{\mathrm{e}}$ siècles, il n'est pas question de "culture" au sens où nous l'entendons de nos jours ${ }^{17}$. Bref, la culture ne saurait devenir mémoire qu'à déplacer profondément ses axes ordinaires.

Le fait que l'on n'hésite pas depuis peut-être une décennie (guère plus) à former un syntagme du type "mémoire culturelle" indique bien l'évolution que Fernand Dumont appelait de ses voux - même si l'on peut aussi s'en méfier et rester prudent devant la dimension volontiers spectaculaire que prend cette improbable alliance. Il n'est pas évident que l'on y décèle l'actualisation d'une sagesse qui réponde aux conditions culturelles de l'universalité en même temps qu'aux valeurs de respect de la tradition, même si tel était justement l'espoir de Fernand Dumont:

Car une sagesse est possible qui, tout en récusant l'absolutisme de la morale, n'est pas pour autant dépourvue de fermeté: une sagesse capable de continuer un héritage sans le répéter, capable de questions neuves sans les escamoter; une sagesse à l'écoute d'autres options et d'autres formules que la sienne, sans se dissoudre dans la platitude du pluralisme naîf. Le règne d'une

16. Fernand Dumont, L'avenir de la mémoire, op. cit., p. 40.

17. Pour un examen de ce déplacement, je renvoie à une monographie à paraître: Le livre avalé: De la littérature entre mémoire et culture ( $x \mathrm{~V}^{P}-\mathrm{xVII} \mathrm{I}^{P}$ siècles). 
telle morale du respect, s'il se pouvait établir, serait la plus haute manifestation de ce processus d'universalisation où nous avons vu la marque irréductible de l'accès aux valeurs ${ }^{18}$.

Comment arrimer ainsi tradition et culture à une croyance au moment où il s'agit toujours d'être, conformément au projet moderne, "sans crédulité envers la légende ${ }^{19}$. Bien sûr, la crédulité n'est que la face sombre et têtue de la croyance. Pourtant est-il possible de s'en débarrasser sans effacer, du coup, la croyance elle-même? Comment croire tout en maintenant une distance critique? Peut-on avoir foi en une tradition qui ne serait plus reçue, mais produite? Et les permanences de la tradition que Dumont souligne dans le discours historique lui-même (héritage des philosophies de l'histoire ou historiographie comme mise à jour des traditions du savoir historique) ne sont-elles autre chose que la dette impayée de l'histoire envers la production du savoir moderne? La baptiser "figure nouvelle de la tradition " ne suffit pas à en fonder la marque mémorielle, sinon à la façon d'une fantasmagorique commémoration de la mémoire elle-même.

Aussi n'est-ce pas un hasard si Dumont alloue aux anciennes traditions "une vision du monde", car c'est déjà les mettre de notre côté et faciliter la récupération de la tradition: une communauté traditionnelle n'a pas de vision du monde, elle a une mémoire. On ne peut en réduire aussi facilement les différences. Heidegger a depuis longtemps souligné que les temps modernes seuls ont inventé des "visions" ou des "conceptions" du monde :

C'est pourquoi les locutions "conception du monde des Temps Modernes " et "conception moderne du monde" disent deux fois la même chose et supposent ce qui n'a jamais été possible auparavant, à savoir une "conception du monde" médiévale et une "conception du monde" antique. Le Monde en tant qu'image conçue ne devient pas, de médiéval, moderne; mais que le Monde comme tel devienne image conçue, voilà qui caractérise et distingue le règne des Temps Modernes. Pour le Moyen Âge, au contraire, l'étant est l'ens creatum, ce qui est créé par le créateur, Dieu personnel agissant en tant que cause suprême ${ }^{20}$.

La difficulté tiendrait donc à ce que Fernand Dumont, habité par l'autorité de la tradition, tenterait de la ramener dans le creuset de la modernité, sans s'apercevoir que la modernité voue ce qui devrait être reçu depuis une extériorité radicale à être produit de façon interne, puisque la tradition suppose un héritage du passé dont chaque présent reçoit les lois, les usages, les pratiques, tandis que la modernité requiert une invention des règles, des valeurs, des significations présentes en fonction d'un futur; or, comment conjoindre d'un même geste hétéronomie et autonomie?

18. Fernand Dumont, Le sort de la culture, op. cit., p. 233.

19. Id., L'avenir de la mémoire, op. cit., p. 58.

20. Voir Martin Heidegger, "L'époque des conceptions du monde ", Cbemins qui ne mènent nulle part, trad. Wolfgang Brokmeier, Paris, Gallimard, 1986 [1949], p. 118. 
Manifestement, nous cherchons aujourd'hui comment repenser ce que nous sommes collectivement, que ce soit sous la forme de nationalismes ou de communautés, d'humanité ou de spiritualité. La mémoire est là qui s'offre. Comment la saisir, comment la faire revivre, alors que nous mettons la liberté au-dessus de l'héritage, la création par-delà la réception?

\section{***}

Fernand Dumont aperçoit bien la difficulté : "dans tous les cas, j’ai affaire à des constructions : la tradition aussi bien que le savoir historique renvoient maintenant à des tâches ${ }^{21}$ ". Comment faire de la tradition une tâche plutôt qu'une dette? Il semble que le fil soit ténu qui permette de le penser. Il existe pourtant. Maintenir la valeur éminente de la dette envers le passé tout en conservant une distance respectueuse vis-à-vis de nos héritages passe par la prise en compte de la blessure.

En donnant à la culture l'envergure d'une distance, d'un entre-deux, d'une plaie, il demeure un respect de la dette en même temps qu'une ouverture au futur. C'était aussi ce que signalait Heidegger, depuis l'élan de la différence ontologique:

Le repli sur la tradition, frelaté d'humilité et de présomption, n'est capable de rien par lui-même, sinon de fuite et d'aveuglement devant l'instant historial. Savoir l'Incalculable, c'est-à-dire le préserver dans sa vérité, l'homme ne le pourra qu'à partir d'un questionnement créateur, puisant dans la vertu d'une authentique méditation. Celle-ci transpose l'homme du futur dans cet Entredeux dans lequel il appartient à l'être cependant qu'au milieu de l'étant il reste un étranger ${ }^{22}$.

Il faudrait réexaminer en ce sens les passages de $\hat{E}$ tre et temps qui traitent du Dasein comme pouvoir-être-en-dette et de son enracinement dans le fait d'être-affecté. J'en indique ici rapidement la teneur: l'appel de la conscience morale (entendue non comme faculté de l'esprit humain mais comme "secteur des phénomènes existentiaux qui constituent l'être du là comme ouverture ${ }^{23_{n}}$ ) est ce qui amène le Dasein au pouvoir-être-propre. Cet appel consiste à faire découvrir comme structure générique du Dasein son être-en-dette (le terme allemand de Schuld signifie aussi bien "faute" que "dette"). Dans la mesure où il est un "être-jeté " dans le monde, le Dasein ne s'est pas lui-même positionné dans l'existence, mais il apparaît comme toujours en retard sur lui-même, puisque le fait d'être ouvert l'ouvre non seulement au monde mais à lui-même; en entendant cet

21. Fernand Dumont, L'avenir de la mémoire, op. cit., p. 59.

22. Martin Heidegger, op. cit., p. 125. Je souligne.

23. Id., Être et temps, trad. François Vezin, Paris, Gallimard, 1985, p. 55 et p. 326. Je rappelle que le Dasein, littéralement "être-là ", peut être compris comme "être-le-là * ainsi que l'indique Heidegger dans une lettre à Jean Beauffret. 
appel, le Dasein fait dès lors de son être-jeté (Geworfenbeit) un projet (Entwurf $)^{24}$. Cette ouverture au temps tient à cet être-affecté dont on peut trouver l'ancienne formulation dans les remarques de Kant par lesquelles j'ai commencé cet article. La "blessure inguérissable", le "déchirement irréductible ", dont traite Fernand Dumont, figurent les traces du temps sur les êtres en même temps que les témoignages qu'ils en portent; ils sont ouverture, appel et dette, signe de cet être-affecté en même temps que témoignage d'un projet.

Tel est bien ce qui permet à Fernand Dumont de faire de la distance qui définit la culture une dette et un appel, ou de la plaie qui signe la tradition une souffrance et un projet. La teneur sociologiquement paradoxale de ces attentes (une culture qui reconnaisse la nécessité des dettes, une tradition qui accepte la distance et le choix) vaut par la position philosophique des enjeux, mais permet aussi de jeter un regard différent sur les réalités quotidiennes et de repérer le travail encore fécond de la tradition dans nos pratiques ou la vertu encore effective d'une culture du projet et de la critique. Est-il étonnant de voir réapparaître chez Fernand Dumont les ressources sereinement théologiques d'une conception de l'histoire et de la tradition qu'il partage, à l'évidence, avec un autre penseur conscient, lui aussi, de leur usage aussi nécessaire que prudent? Pour Walter Benjamin, "la remémoration peut transformer ce qui est inachevé (le bonheur) en quelque chose d'achevé et ce qui est achevé (la souffrance) en quelque chose d'inachevé. C'est de la théologie; mais nous faisons, dans la remémoration, une expérience qui nous interdit de concevoir l'histoire de façon fondamentalement athéologique, même si nous n'avons pas, pour autant, le droit d'essayer de l'écrire avec des concepts immédiatement théologiques ${ }^{25}$." On conçoit, du coup, la possibilité de distinguer deux usages de la tradition: l'un qui reconduit le poids de ce qui est transmis, avec son lot de préjugés et d'inhibitions, jusque dans la tradition du nouveau; l'autre qui refait l'expérience du legs et de la dette, pour mieux y découvrir la nouveauté de la tradition. D'un côté, on souscrira d'avance à la célébration d'un passé continu et étanche qui ne nous apprend plus rien; de l'autre, on deviendra attentif à la discontinuité de chaque situation dans laquelle pénètrent les échardes de l'Autrefois. En ce sens, Fernand Dumont n'aurait probablement pas désavoué cette remarque de Walter Benjamin :

De quel péril les phénomènes sont-ils sauvés? Pas seulement, et pas principalement du discrédit et du mépris dans lesquels ils sont tombés, mais de la catastrophe que représente une certaine façon de les transmettre en les "célébrant " comme "patrimoine". - Ils sont sauvés lorsqu'on met en

24. Ibid., p. 58-59.

25. Walter Benjamin, Paris, capitale du xIX siècle. Le livre des Passages, trad. Jean Lacoste, Paris, Cerf, 1993, p. 489. 
évidence chez eux la fêlure. - Il y a une tradition qui est une catastrophe ${ }^{26}$.

La pensée de Fernand Dumont est encore, par elle-même, un exemple de cette intrication possible, souhaitée, entre mémoire et culture, puisque l'entente de la tradition repose, chez lui, sur le constant souci d'une transcendance dans l'histoire humaine, puisque l'écoute de la culture suppose, pour lui, une ouverture aux phénomènes douloureux du monde. Par delà ou en deçà des passages aux horizons théologiques, cette transcendance vaut moins aujourd'hui pour des échappées attendues hors du monde des hommes que pour un juste retour des choses. Contrairement, donc, à ce que l'on a souvent pensé, la tradition ne reconduit pas simplement les valeurs et les pratiques de jadis, elle peut aussi introduire dans la culture des hommes et des femmes le caractère explosif du passé. Dans une notice préparatoire à ses Thèses sur le concept d'bistoire, Walter Benjamin oppose le continuum historique des oppresseurs à la discontinuité de la tradition qui relève des opprimés. Mais il existe encore une continuité secrète de la tradition, comme le remarque Michael Löwy: "à l'image de l'explosion qui doit briser le continuum de l'oppression correspond, dans le domaine de la tradition des opprimés, la métaphore du tissage : [...] il faut tisser, dans la trame du présent, les fils de la tradition qui ont été perdus pendant des siècles ${ }^{27}$ ". Sans que Fernand Dumont ni Walter Benjamin l'aient jamais vraiment exploité, on retrouve chez eux un souci de l'antique kairós qui désigne aussi bien l'occasion, la règle, le contrôle stratégique, que la décision ou la brièveté, le continu ou la coupure, et dont l'origine se trouve dans un élément du métier à tisser, le kaî$\operatorname{ros}^{28}$.

26. Ibid., p. 490-491. On pourrait rapprocher aussi cette conception de celle qui anime, chez Jacques Derrida, la remémoration de la terreur politique: "Car il y a des situations, des expériences, des sujets qui sont justement en situation (mais qu'est-ce que situer veut dire dans ce cas?) d'en témoigner exemplairement. Cette exemplarité ne se réduit plus simplement à celle de l'exemple dans une série. Ce serait plutôt l'exemplarité remarquable et remarquante - qui donne à lire de façon plus fulgurante, intense, voire traumatique, la vérité d'une nécessité universelle. La structure apparaît dans l'expérience de la blessure. (Jacques Derrida, Le monolinguisme de l'autre, ou la protbèse d'origine, Paris, Galilée, 1996, p. 48-49)

27. Michael Löwy, Walter Benjamin. Avertissement d'incendie. Une lecture des thèses "Sur le concept d'bistoire „, Paris, Presses universitaires de France, 2001, p. 104.

28. Kaîros désigne un élément du métier à tisser: d'un diamètre minime, il s'agirait d'une sorte de "tresse régulatrice", double cordelette (l'une pour la rangée des fils pairs, l'autre pour la rangée des fils impairs) formée d'une succession d'anneaux dans lesquels passe un petit nombre de fils variant avec le tissu à confectionner. Il y aurait ainsi, selon Bernard Gallet, quatre grandes fonctions du kaîros qui conduiraient aux divers usages figurés du kairós: 1) du fil conducteur, qui maintient et rend solidaires tous les autres fils, qui agit donc sur eux, on obtiendrait les sens de prise, de moyen, de contrôle stratégique; 2) du fil régulateur, qui ordonne les fils de la chaîne, on tirerait les sens de règle, de juste mesure, mais aussi de limite, de brièveté (puisqu'il a une longueur égale à la largeur du métier, tandis que les fils de chaîne ou de trame sont beaucoup plus longs), voire d'avantage (puisqu'on y gagne un tissu de confection impeccable); 3) du 
L'homme ne devrait pas oublier qu'il est ainsi un (modeste) artisan du temps. Le tissage de chaque trame du présent ne doit pas seulement exhiber les conquêtes et la continuité de l'histoire, mais aussi, peut-être surtout, les discontinuités de l'oppression. Les enjeux politiques de la tradition, de la mémoire, de la culture sont pour Fernand Dumont des enjeux sociaux et l'usage de la "blessure ", la marque explicite des opprimés. Ce n'est pas seulement le pliage d'un Dieu au format humain qui fait la particularité du christianisme qu'a tant pratiqué Fernand Dumont, mais aussi sa souffrance avouée. Ainsi que le souligne, de son côté, Pierre Legendre:

Voir la blessure du Christ, la célébrer, fut pour les sociétés du christianisme médiéval l'équivalent de dire et penser les fondements, la scène de l'homme et du monde, à l'analogue de ce qui est dit et pensé, dans la culture sécularisée ultramoderne, par les interprètes de l'énigme ${ }^{29}$.

Le fondement n'a d'autre lieu que celui de sa réitération en expérience, de sa situation au creux du temps, variable et exemplaire, allouant ainsi aux instants la puissance d'une énigme. Il faut à l'homme un milieu où habiter, quelque chose qu'il peut achever, mais il lui faut aussi un univers qui l'habite et le rende, dès lors, inachevable.

fil entrelacé, qui vient rencontrer chacun des fils de la chaîne à angle droit, on aurait l'idée de conjoncture, de coïncidence, de moment propice et à nouveau d'avantage; 4) du fil séparateur, qui isole les deux nappes de fils pairs et de fils impairs, et, dans chacune des nappes, qui sépare les fils pour qu'ils ne s'emmêlent pas, proviendraient les idées de choix, de jugement et de décision, de ce qui tranche entre des solutions. La teneủr temporelle et oisive du kairós prend toute son ampleur de l'expérience quotidienne du travail artisanal et de l'espace spécifique du métier à tisser. Voir Monique Trédé, Kairos: l'à-propos et l'occasion. Le mot et la notion d'Homère à la fin du $N^{e}$ siècle avant J.C., Paris, Klincksieck, 1992; Bernard Gallet, Recherches sur kairos et l'ambiguité dans la poésie de Pindare, Bordeaux, Presses universitaires de Bordeaux, 1990.

29. Pierre Legendre, Dieu au miroir: Etude sur l'institution des images. Leçons III, Paris, Fayard, 1994, p. 27. 